\title{
USP General Chapter $<825>$ Impact on Nuclear Medicine Technology Practice
}

\author{
George H. Hinkle, RPh, BCNP \\ Radiation Safety Office, Environmental Health and Safety, Ohio State University, Columbus, Ohio
}

\begin{abstract}
CE credit: For CE credit, you can access the test for this article, as well as additional JNMT CE tests, online at https://www.snmmilearningcenter.org. Complete the test online no later than June 2023. Your online test will be scored immediately. You may make 3 attempts to pass the test and must answer $80 \%$ of the questions correctly to receive $1.0 \mathrm{CEH}$ (Continuing Education Hour) credit. SNMMI members will have their CEH credit added to their VOICE transcript automatically; nonmembers will be able to print out a CE certificate upon successfully completing the test. The online test is free to SNMMI members; nonmembers must pay $\$ 15.00$ by credit card when logging onto the website to take the test.
\end{abstract}

\begin{abstract}
U.S. Pharmacopeia (USP) general chapter $<825>$, "Radiopharmaceuticals: Preparation, Compounding, Dispensing, and Repackaging," is a new standard proposed to provide minimum requirements for the preparation, compounding, dispensing, and repackaging of sterile and nonsterile radiopharmaceuticals. This new standard represents endeavors on the part of the USP to respond to appeals by nuclear medicine professionals to move beyond a minimal supplement to USP $<797>$ and provide policies specific to radiopharmaceuticals. USP $<825>$ provides nuclear pharmacies and nuclear medicine departments in hospitals and clinics with the benchmarks to assess current practice activities and integrate needed changes to meet regulatory and accreditation audit reviews. This continuing education article focuses on components of USP $<825>$ specific to the nuclear medicine technologist for a better understanding of obligations when preparing sterile radiopharmaceuticals for clinical use.
\end{abstract}

Key Words: USP <825>; radiopharmaceutical compounding; sterile radiopharmaceutical; USP standards

J Nucl Med Technol 2020; 48:106-113

DOI: $10.2967 /$ jnmt.120.243378

$\mathbf{R}$ adiopharmaceuticals, or radioactive drugs, are controlled by international, federal, state, and even local agencies. The Nuclear Regulatory Commission or contracted agreement-state agencies regulate radiopharmaceuticals to ensure the safe use of radioactive materials. The Department of Transportation and the Environmental Protection Agency control the shipment and disposal of radioactive drugs. Radiopharmaceuticals are included in the category of prescription drugs, with the Food and Drug Administration

Received Feb. 25, 2020; revision accepted Apr. 22, 2020.

For correspondence or reprints contact: George H. Hinkle, RPh, BCNP, Radiation Safety Office, Environmental Health and Safety, Ohio State University, 1314 Kinnear Rd., Columbus, OH 43212.

E-mail: hinkle.5@osu.edu

COPYRIGHT @ 2020 by the Society of Nuclear Medicine and Molecular Imaging.
(FDA) responsible for manufacturing and ultimate patient safety. State medical and pharmacy boards require compliance with standards to ensure that providing prescription drugs to patients produces the best outcomes.

Health-care professionals involved in the use of radiopharmaceuticals must conform with a myriad of regulations, guidelines, and standards in a variety of practice settings to help ensure patient safety and protect the general population and the environment. Compliance is often secured through the publication and expected observation of practice standards, policies, procedures, and rules.

\section{U.S. PHARMACOPEIA (USP)}

Founded in 1820, the USP is a nongovernment organization that provides standards of identity, strength, quality, purity, and labeling for medications, pharmaceutical excipients or additives, and dietary supplements. Originally formed to confirm the safe use of drugs through the publication of formulas, quality tests, and identity tests, the USP evolved over time and, through interactions with the FDA, began to develop and publish standards that describe storage, dispensing, packaging, preparation, and specifications for conditions and practices for pharmaceutical compounding. USP publications, called general chapters, include both informational and enforceable standards. The USP has no authorization to conduct inspections. State pharmacy board inspectors and institutional accreditation agency surveyors often use USP standards for practice evaluation. USP standards are often cited in regulatory and reference publications.

\section{THE USP AND RADIOPHARMACEUTICALS}

In 1995, the USP provided information and recommendations for compounding sterile drug products in general chapter $<1206>$. Voluntary compliance with guidelines in USP $<1206>$ proved inadequate, with large numbers of patients reportedly having infections caused by contaminated compounded drug products. In 2004, the USP published general 
chapter $<797>$, "Pharmaceutical Compounding: Sterile Preparations," which specifies that sterile compounding applies to all preadministration manipulations (preparation, storage, and transport) of compounded sterile preparations, including radiopharmaceuticals ( 1 ). Although this publication was generally ignored, nuclear medicine professionals were provided standards describing the preparation of sterile drugs, including radiopharmaceuticals, for patient use. Although radiopharmaceuticals were not explicitly mentioned in the original publication, the intent was that USP $<797>$ would apply to all sterile compounding activities. The first revision of USP $<797>$, in 2008 , included a section on radiopharmaceuticals as compounded sterile preparations; this revision contained standards for radiopharmaceuticals and nonradioactive drugs (1). Most hospitals and clinics outsource the compounding of radioactive drugs to commercial nuclear pharmacies. However, the preparation of radiopharmaceutical dosages for emergency procedures and other on-site manipulations involving radioactive drugs has been under the guidance of the USP since 1995. The Joint Commission and other accreditation bodies, state boards of pharmacy, and the Centers for Medicare and Medicaid Services will act to enforce the standards. Sterile compounding standards in USP general chapters apply to all places and all health-care personnel involved in the preparation, storage, manipulation, and transportation of compounded sterile preparations. With regard to radiopharmaceuticals, nuclear medicine technologists and nuclear medicine facilities are included among the healthcare personnel to whom these standards apply. Along with following the regulations and accreditation standards, nuclear medicine technologists are directed to comply with USP standards on the preparation of sterile radiopharmaceuticals. USP standards are cited in published practice and performance standards (2) for the cleansing and garbing protocols to be used before staff enters clean areas, as well as for protocols for aseptic technique in vial puncture procedures, for establishing beyond-use dates (BUDs) or beyond-use times, and for proper storage of radiopharmaceutical kits, radionuclide generators, and prepared radiopharmaceuticals.

\section{COMPLIANCE PROBLEMS WITH USP $<797>$ AND RADIOPHARMACEUTICALS AS COMPOUNDED STERILE DRUGS}

Nuclear medicine professionals directed to follow USP $<797>$ standards were faced with challenges unique to the preparation of radiopharmaceuticals. The primary issue involved merging radiation safety standards with the aseptic handling scenarios described in USP $<797>$. Established radiation safety practices requiring lining of workspace surfaces with absorbent paper, use of syringe and vial shields, positioning of lead-glass L-shields in front of laminar flow workstations, and placement of radiation-measuring instrumentation (dose calibrators) in the same environment where sterile compounding takes place largely contradicted USP guidance for the creation of a sterile workspace. Lack of specific guidance on sterile compounding of radioactive drugs led to confusion among nuclear medicine professionals on how to interpret the standards, as well as confusion among inspectors who were using the USP $<797>$ standards for guidance. Guidance for sterile compounding of radioactive drugs in USP $<797>$ was largely ignored by nuclear medicine professionals until a failed inspection left the facility with a list of required modifications to practice. Nuclear medicine professionals, as well as regulators and inspectors, recognized the need for radiopharmaceutical specific standards (3).

\section{USP $<825>$ SCOPE, GOALS, AND OBJECTIVES}

During the 2016 revision of USP $<797>$, nuclear medicine professionals submitted over 1,500 comments, with most including examples of the failure of USP $<797>$ to meet the requirements of radiation safety while attempting to conform with the standards associated with sterile compounding of radioactive drugs. A Society of Nuclear Medicine and Molecular Imaging publication identified practices in the preparation of radiopharmaceuticals that are different from the common sterile compounding activities associated with nonradioactive drugs and concluded with a list of recommendations that establish criteria for preparation of radiopharmaceuticals that follow aseptic guidelines while meeting as-low-as-reasonably-achievable (ALARA) principles (3). Eventually, the USP standards committee was convinced that a distinct set of specifications for radiopharmaceuticals was needed. This determination led to the establishment of an expert panel on radiopharmaceuticals to develop the model that would provide guidance for the activities associated with the sterile compounding of radiopharmaceuticals. USP general chapter $<825>$, "Radiopharmaceuticals: Preparation, Compounding, Dispensing, and Repackaging," provides standards for the preparation, compounding, dispensing, and repackaging of sterile and nonsterile radiopharmaceuticals included in the practice of pharmacy and medicine (4). It applies to all practice settings where these activities involving radiopharmaceuticals occur, including nuclear pharmacies, nuclear cardiology imaging clinics, and nuclear medicine departments of hospitals. It applies to sterile and nonsterile radiopharmaceuticals and to direct infusion radionuclide generators and devices. It applies to all individuals involved in the preparation, compounding, dispensing, and repackaging of radiopharmaceuticals, including pharmacists and physicians who hold authorized user regulatory status and those working under their supervision, such as pharmacy technicians, nuclear medicine technologists, residents, and students or trainees. USP general chapter $<823>$, "Radiopharmaceuticals for PET: Compounding," supersedes USP $<797>$ and $<825>$ for guidance involving PET radiopharmaceuticals. On release of a PET radiopharmaceutical as a finished drug product from the commercial manufacturing facility, further handling, manipulation of unit doses from a vial, and use of the product are considered compounding activities and subject to standards in USP 
$<797>$ and $<825>$. Nuclear medicine departments and nuclear cardiology imaging clinics involved in administering to patients unit-dose radiopharmaceuticals received from a nuclear pharmacy, with no additional manipulation after delivery, are not subject to USP standards other than to ensure that the nuclear pharmacy outsourcing facility is compliant (4).

USP $<825>$ models USP $<797>$ with needed modifications. USP $<825>$ includes content to provide minimum standards for the preparation, compounding, dispensing, and repackaging of sterile and nonsterile radiopharmaceuticals. The USP $<825>$ standards describing facilities and engineering controls, personnel qualifications, training and hygiene, cleaning and disinfecting, and air and surface monitoring mostly parallel those found in USP $<797>$ (4). In the sterile compounding, preparation, dispensing, and repackaging of radiopharmaceuticals in a nuclear pharmacy setting, USP $<825>$ standards for those activities mostly correspond to those in USP $<797>$.

\section{USP $<825>$ RADIOPHARMACEUTICAL-SPECIFIC STANDARDS}

The differences found in USP $<825>$ reflect the unique properties of radioactive drugs and emphasize the importance of following good radiation safety practices while being attentive to the time element for the clinical use of radiopharmaceuticals and balancing with aseptic handling practices. USP $<825>$ addresses the failure of USP $<797>$ to include radiation safety considerations. Placement of absorbent pads in hoods, handling of sharps during radiopharmaceutical compounding, and positioning within hoods the radiation-measuring instrumentation needed during compounding activities were specifically adopted in the new standard (4).

\section{RADIOPHARMACEUTICAL PREPARATION OR COMPOUNDING}

USP $<825>$ clearly differentiates radiopharmaceutical preparation from compounding. Preparation is the customary radiolabeling procedure in which the radionuclide is combined with the chemical carrier designed to deliver the radioactivity to the organ of interest or the target. A collection of actions, including mixing, combining, diluting, or reconstituting, can be used to prepare the nonsterile or sterile radiopharmaceutical. Except for minor deviations, preparation must be done according to directions found in the FDA-approved labeling for the conventionally manufactured radiopharmaceutical kit and radionuclide. Unless intended for immediate use, sterile radiopharmaceutical preparation must be completed in an International Organization for Standardization (ISO)-classified area or device to ensure that the minimum environmental standard is met (Table 1). The required primary engineering control (PEC), secondary engineering control, and manipulations involved in the radiopharmaceutical preparation are used to determine the BUD for the final preparation (4). Along with environmental controls, aseptic handling practices to maintain sterility must be followed by personnel, including prior aseptic procedure training or qualification, hand hygiene, and garbing. The final preparation must be assessed to ensure that it will meet the quality and purity specifications throughout the assigned BUD. Depending on the radiopharmaceutical, this assessment may include testing for radionuclide purity, radiochemical purity, and chemical purity, as well as physical properties (4).

Compounding is recognized as a major alteration to the FDA-approved directions for preparation or radioactive drug formulation from bulk drug substances and radionuclides. Established written procedures for compounding radiopharmaceuticals must be in place, with records of the materials used and the testing results for each finished product. Sterile compounding must follow aseptic procedures and be completed in an ISO class 5 PEC (Table 1). USP $<825>$ prohibits the compounding of conventionally marketed, FDA-approved radiopharmaceuticals except for clinically relevant changes requested for a specific patient by a licensed physician. Compounding radiopharmaceuticals removed from commercial distribution can proceed only as part of an investigational study approved by the institution's review board (4). When nonsterile components or bulk drug substances for sterile compounding are used, a final sterilization procedure with testing for sterility and freedom from pyrogens must be completed. Additional tests for purity, identity, quality, chemical stability, radiochemical stability, and $\mathrm{pH}$, as well as changes to any of these parameters, must be established for assignment of the BUD to the final product.

\section{PREPARATION WITH MINOR DEVIATIONS}

On occasion, circumstances not addressed in FDA-approved labeling require variation from the manufacturer's preparation instructions. Patient-care requisites, or variations in the

TABLE 1

ISO Classification of Particulate Matter in Air and PEC or Secondary Engineering Controls

\begin{tabular}{lll}
\hline ISO class & Particle count $/ \mathrm{m}^{3}$ of air & PEC or secondary engineering controls \\
\hline 3 & 35.2 & \\
4 & 352 & PEC \\
5 & 3,520 & \\
6 & 35,200 & Buffer area, secondary engineering control \\
7 & 352,000 & Anteroom, radionuclide generator elution \\
8 & $3,520,000$ & Segregated radiopharmaceutical processing area \\
Unclassified & Ambient room air & \\
\hline
\end{tabular}


availability or chemical or physical properties of radiolabeling components, may require a minor deviation from the routine radiopharmaceutical preparation. Variations may involve differences in the specific activity of the final preparation or proportions of the components of the radiopharmaceutical kit used in the formulation. Examples include a change in the FDA-approved step-by-step labeling instructions, a variation in the quantity of radioactivity, or a variation in the final volume of the preparation. Such variations are common when the nuclear medicine technologist prepares a ${ }^{99 \mathrm{~m}} \mathrm{Tc}$-aggregated albumin vial to meet a physician's request for fewer particles for a specific patient. Minor deviations may involve the use of alternative devices or equipment (different sizes of needle or syringe, different shielding materials, use of a heating block instead of a water bath), use of quality control testing other than that described in the prescribing information, and filtration of ${ }^{99 \mathrm{~m}} \mathrm{Tc}$ sulfur colloid for lymphatic mapping procedures. However, alterations from the manufacturer's instructions must maintain the same ingredients and must not include the addition of extra components (4).

\section{RADIOPHARMACEUTICAL PROCESSING ENVIRONMENT}

The USP $<825>$ standards model those in USP $<797>$ for design and layout and the required engineering controls for the sterile radiopharmaceutical processing. With the understanding that most microbial contaminants are carried through the air on particulates, following the guidelines of USP $<825>$ ensures that the sterile radiopharmaceutical preparation and compounding procedures are completed in areas and under engineering controls that meet air quality standards for the activities to be completed, thereby reducing the likelihood of contamination. Along with control of temperature and humidity, the use of nonshedding building materials on the surfaces of walls, floors, and ceilings; planning of personnel traffic; general cleaning; and manipulation of the air currents from the heating, ventilation, and air conditioning system or opened doors, selection and placement of PEC is critical for establishment of an environment favorable for sterile radiopharmaceutical processing. A PEC is a laminar flow hood using a unidirectional, high-efficiency particulate air-filtered airflow to create an ISO class 5 or better workspace. When used for preparation, for preparation with minor deviations, for dispensing, or for repackag- ing of sterile radiopharmaceuticals, the ISO-class PEC may be located in an unclassified segregated radiopharmaceutical processing area with low traffic and away from air currents that may disrupt the laminar airflow inside the hood. The segregated radiopharmaceutical processing area does not require filtered air along with air monitoring. If used in the compounding of sterile radiopharmaceuticals, the PEC must be located within an ISO class 7 or better buffer area, along with an ISO class 8 or better anteroom (4).

\section{TRAINING AND QUALIFICATION OF PERSONNEL}

Sterile compounding or preparation activities outside immediate-use practice require prior completion of training on policies and standard operating procedures established by the authorized nuclear pharmacist or authorized user physician. In addition, training on aseptic handling and blood-borne pathogens, if appropriate, is required. Before conducting aseptic techniques involved with radiopharmaceutical compounding beyond immediate use or radiolabeled blood component preparation, personnel must prove competency. Successful completion of the job functions listed in Table 2 under the observation of a designated person is required for initial qualification and at 12-mo intervals, depending on sterile compounding responsibilities (4).

\section{HIGHLIGHTS OF USP $<825>$ FOR NUCLEAR MEDICINE TECHNOLOGISTS}

Compliance with USP $<825>$ standards is determined by the practice activities of the nuclear medicine technologist. Most USP $<825>$ standards will not apply to a nuclear medicine practice that outsources the preparation of radiopharmaceuticals and receives patient dosages ready for administration. For nuclear medicine departments using radionuclide generators or bulk radionuclide material and radiopharmaceutical kits for the preparation of multidose vials, USP $<825>$ provides benchmarks for those activities that are subject to audit or inspection. For facilities involved in radiopharmaceutical preparations only under the immediate-use criteria, less rigorous area and personnel standards are applied for final preparations, with more austere requirements for storage and administration, as seen in Table 3. Immediate-use provisions allow for benchtop preparation but require that a single dose be prepared for a single patient within $1 \mathrm{~h}$ after the formulation begins, as

TABLE 2

Aseptic Qualifications

\begin{tabular}{|c|c|c|}
\hline Activity & Evaluation & Timing for requalification \\
\hline Aseptic technique training & Documented assessment & Initially only \\
\hline Garbing and hand hygiene & $\begin{array}{l}\text { Observation for policy or standard-operating-procedure } \\
\text { compliance }\end{array}$ & Every 12 mo \\
\hline PEC cleaning or disinfecting & $\begin{array}{l}\text { Observation for policy or standard-operating-procedure } \\
\text { compliance }\end{array}$ & Every 12 mo \\
\hline Gloved fingertip or thumb sampling & Media-fill testing of gloved fingertip and thumb sampling & Every $12 \mathrm{mo}$ \\
\hline Media-fill testing of aseptic procedures & Media-fill testing of radiopharmaceutical manipulations & Every 12 mo \\
\hline
\end{tabular}


TABLE 3

Radiopharmaceutical Preparation Requirements

\begin{tabular}{lll}
\hline \multicolumn{1}{c}{ Function } & \multicolumn{1}{c}{ Immediate use } & \multicolumn{1}{c}{ Beyond immediate use } \\
\hline Preparation area & Hot lab with ambient room air & $\begin{array}{c}\text { Segregated radiopharmaceutical } \\
\text { processing area or clean room }\end{array}$ \\
Cleaning or disinfection of preparation area & Required & Required \\
Quality control testing of final product & Required & Required \\
Garbing and hand hygiene & Required (clean lab coat) & Required (full garbing) \\
Aseptic technique training & Required & Required \\
PEC (hood) use & Not required & Required \\
Gloved fingertip or thumb sampling test & Not required & Required \\
Media-fill test & Not required & Required \\
Aseptic manipulation competency test & Not required & Required \\
\hline
\end{tabular}

well as requiring that any remaining material be discarded, since the radioactive drug was completed in a nonsterile environment.

\section{INTERVENTIONAL AGENTS AND ADJUNCT MEDICATIONS}

USP $<797>$ placed limitations on the preparation of nonradioactive drugs for use as interventional agents in diagnostic and therapeutic studies conducted in nuclear medicine. USP $<825>$ does not apply to nonradioactive drugs such as amino acid preparations, morphine, sincalide, furosemide, and regadenoson. Language in USP $<825>$ specifically references adherence to standards in USP $<797>$ when nonradioactive drugs are prepared for patient use. USP $<797>$ standards for immediate use must be followed, including aseptic-technique preparation, singlepatient use, a time limit for patient administration, and labeling requirements (4).

\section{FOLLOWING ALARA PRINCIPLES}

USP $<797>$ did not address issues of radiation safety and the ALARA benchmarks. Observance of the established radiation safety practices of time, distance, shielding, and contamination control while maintaining USP $<797>$ specifications during radiopharmaceutical sterile compounding procedures was not possible. USP <825> addresses considerations for radiation safety, including observation of ALARA practices, noting the need for multiple hand movements into and out of the hood during aseptic handling procedures to limit the time of exposure; use of remote handling tools to increase the distance from vials and syringes, use of vial and syringe shielding and an L-block in the front of the hood to reduce exposure during radioactive drug preparation; and lining of the surface of the hood with absorbent pads to limit spill contamination. The requirement for inside-the-hood placement of the dose calibrator, disposable sharp container, and other instrumentation required during the radioactive drug preparation and dispensing functions was an effort to balance the aseptic handling process with established radiation safety practices. Addressing the primary reason for failure to comply with USP $<797>$ requirements was a focused objective of USP $<825>$.
Before USP $<825>$, sterile compounding guidelines for immediate use limiting the number of needle entries through the vial septum to no more than 2 were not compatible with ${ }^{99 m}$ Tc-labeled red blood cell preparation, which requires 5 needle punctures (5). USP $<825>$ includes a section entitled, "Preparation of Radiolabeled Red Blood Cells for Immediate Use," containing a description of dedicated space and equipment for the red blood cell radiolabeling process, cleaning and disinfecting procedures for the space and equipment, as well as hand hygiene and garbing requirements. Although immediate use lists a BUD of $1 \mathrm{~h}$, there are no restrictions on the number of needle punctures during the radiolabeling process (4). Following standards established in USP $<825>$, the nuclear medicine technologist can prepare ${ }^{99 \mathrm{~m}}$ Tc-labeled red blood cells for a patient on a lab bench in a hot lab using recognized ALARA principles and aseptic technique.

\section{THE NUCLEAR MEDICINE HOT LAB}

Recognized as an area for manipulations of radioactive materials, including sterile preparation of radiopharmaceuticals for immediate use, the nuclear medicine hot lab is defined in USP $<825>$ as an unclassified (ambient or unfiltered air) radiopharmaceutical processing area located within a hospital or clinical site that is appropriate only for immediate-use radiopharmaceuticals. USP $<825>$ recognizes the need for radioactive drugs to be prepared outside the sterile environment created in a hood within a clean room with high-efficiency particulate air-filtered air. The nuclear medicine technologist is responsible for observing hot lab restrictions, include limiting traffic in the area during radiopharmaceutical preparation as well as separating the space from patient care and nonsterile activities such as radiolabeling meals for gastric emptying studies. Before sterile radiopharmaceutical preparation in a hot lab, the nuclear medicine technologist must follow strict aseptic technique, including hand hygiene and garbing as well as cleaning and disinfecting of the area (lab bench) where the drug preparation will occur (4). The hot lab must be clean, and all clinical activities involving patient care must be stopped during the sterile radiopharmaceutical preparation. 


\section{PREPARATION OF RADIOPHARMACEUTICALS FOR IMMEDIATE USE}

Although USP $<797>$ provided strategies for the preparation of radiopharmaceutical doses for immediate use, the criterion included restrictions that were subject to interpretation and often not amenable to radioactive drug preparation and dispensing. USP $<825>$ includes a description of the preparation and dispensing of sterile radiopharmaceuticals for immediate use in a patient care setting (4). Preparation components must be sterile FDA-approved kits, the finished product must be limited to use for a single patient, and the finished product must be administered within $1 \mathrm{~h}$ of the first vial puncture that started the radiolabeling procedure. Along with radiopharmaceutical kit preparation, manipulations of unit doses (needle changes, adjustments of the radioactivity dose or volume), dispensing of a single dose from a prepared vial, dose pooling (combining doses to meet a single-patient need), and addition of a nonradioactive pharmaceutical to a unit dose (e.g., adding lidocaine to a ${ }^{99 \mathrm{~m}} \mathrm{Tc}$-radiopharmaceutical for lymphatic mapping or sentinel lymph node imaging) are allowed under immediateuse provisions. Manipulation of a single dose to meet the needs of multiple patients (dose splitting) may not be done under immediate-use standards (4). Missing from the immediate-use provisions of USP $<797>$ are guidelines - provided in USP $<825>$-for the qualifications for personnel who prepare these sterile drugs; USP $<825>$ incorporates guidance on completing a sterile radiopharmaceutical preparation, including directions for hand hygiene, gloving, garbing, and the required aseptic technique, as well as blood borne pathogen training (4). Table 4 summarizes the conditions that must be met for sterile preparation of radiopharmaceuticals for immediate use.

\section{QUALITY CONTROL OF RADIOPHARMACEUTICALS}

Completion of a quality assessment, including the physical appearance, radiochemical purity, and radionuclidic purity of radiopharmaceuticals prepared or compounded on site, is no longer optional. Determination of the quality and purity of prepared radiopharmaceuticals, as appropriate, is mentioned in the preparation and documentation sections of USP $<825>$. Applicable quality testing - the need for which is specifically mentioned for radiopharmaceuticals that are prepared with minor deviations from the manufactured kit directions-is mandatory for validating the radiochemical purity of the final product through to the BUD (4). The nuclear medicine technologist is responsible for complying with this standard, as is supported by published practice standards as well (6).

\section{RADIOLABELING OF BLOOD COMPONENTS}

USP $<797>$ contained no sterile compounding guidance for the radiolabeling of blood components, including leukocytes, platelets, and stem cells. To provide direction for this important area of radioactive materials preparation, USP $<825>$ includes guidelines for the preparation of radiolabeled blood components (4). There are strict requirements for the location and procedure to prevent microbiologic contamination of the blood product or of the area, equipment, or instruments used during the radiolabeling procedure. Radiolabeling of blood components other than red blood cells is not included in the immediate-use category of USP $<825>$ and requires strict measures for the creation of a sterile preparation area. Standards include instructions for complete separation of areas where blood components are handled and the use of an ISO class 5 hood in an ISO class 7 buffer area to maintain the air quality of the radiolabeling space. Also, cleaning or disinfecting procedures for hoods, equipment, and instruments and instructions for hand hygiene, garbing, and prior aseptic training or qualification of staff are addressed. Directives for labeling of tubes, syringes, and other supplies with patient identifiers and a time limit of $6 \mathrm{~h}$ after blood collection for administration of the finished radiolabeled product are contained in the specifications. Blood components from a single patient only are allowed in the PEC during the radiolabeling procedure. Cleaning and disinfection of the hood and reusable equipment, including the dose calibrator liner and dipper,

TABLE 4

USP $<825>$ Immediate-Use Sterile-Radiopharmaceutical Preparation Requirements

\begin{tabular}{|c|c|}
\hline Parameter & Requirement \\
\hline Area (hot lab) & Ambient (unfiltered) room air with no engineering controls (hood) \\
\hline Area restrictions & $\begin{array}{l}\text { Separate from nonsterile compounding and patient care activities during preparation; } \\
\text { limited traffic during preparation }\end{array}$ \\
\hline Preparation (FDA-approved kit) & Sterile, conventionally manufactured radiopharmaceutical kits \\
\hline Preparation restrictions & $\begin{array}{l}\text { Single dose for single patient administered within } 1 \mathrm{~h} \text { of start of preparation; remaining } \\
\text { material discarded }\end{array}$ \\
\hline Personnel & Training on blood-borne pathogen and aseptic handling techniques \\
\hline Personnel restrictions & Adherence to hand hygiene, garbing, and gloving procedures, as in section 4.4 (4) \\
\hline Activities & $\begin{array}{l}\text { Unit-dose manipulations allowed (needle changes, adjusting of radioactivity or volume); } \\
\text { dispensing single dose from vial; dose pooling for single patient dose; addition of } \\
\text { nonradioactive, sterile, commercially manufactured pharmaceutical to unit dose }\end{array}$ \\
\hline Activity restrictions & $\begin{array}{l}\text { Radiolabeling of leukocytes or platelets and splitting of single-unit dose for more } \\
\text { than } 1 \text { patient are not allowed }\end{array}$ \\
\hline
\end{tabular}


must be completed before use for another radiolabeling procedure (4).

\section{PREPARATION OF NONSTERILE RADIOACTIVE PRODUCTS}

Hospitals and clinics that rely on commercial nuclear pharmacies for the preparation and delivery of unit-dose radiopharmaceuticals for most of their patient needs often become involved in the preparation of nonsterile radioactive products, usually to meet a specific need for a single patient. Compounding of nonsterile radiopharmaceuticals is described in USP $<825>$ as the combining, mixing, diluting, pooling, reconstituting, or otherwise altering of a drug to create a nonsterile radiopharmaceutical (4). Included in this category is changing from a sterile intravenous dosage form to an oral dosage form, changing from an oral capsule to a liquid, preparation of an oral capsule from a liquid, and radiolabeling of a food substance for oral administration. A description of the area designated for nonsterile compounding and of the required records and materials used for nonsterile radiopharmaceutical compounding is included in the USP $<825>$ standards. Separation of the area and activities involved in compounding nonsterile radiopharmaceuticals from those used in the preparation of sterile radiopharmaceuticals is addressed in the standard. The area designated for nonsterile compounding must be clean, uncluttered, free of patient care activities, and separate from the area designated for sterile radiopharmaceutical preparations.

\section{BUD ASSIGNMENT}

USP $<825>$ provides information on establishing the assigned time beyond which the compounded radiopharmaceutical must not be administered. Closely associated with the risk level of the prepared radioactive drug, the BUD is dependent on the preparation conditions, the known or measured quality and purity parameters, and the storage environments. BUD is determined with consideration of the manipulations involved in the radiopharmaceutical preparation, maintenance of sterility, radiochemical and radionuclide purity, the age of the radionuclide generator eluate used in the radiopharmaceutical preparation, the specific activity, and the preparation conditions. The established BUDs are $6 \mathrm{~h}$ for radiolabeled blood components, $1 \mathrm{~h}$ for ${ }^{99 \mathrm{~m}} \mathrm{Tc}-$ labeled red blood cells for immediate use, $1 \mathrm{~h}$ for sterile radiopharmaceuticals for immediate use, $10 \mathrm{~h}$ for direct infusion systems, and $24 \mathrm{~h}$ for preparing and dispensing radiopharmaceuticals prepared under classic sterile conditions (4).

\section{DIRECT INFUSION SYSTEMS}

USP $<825>$ provides guidance on maintaining the sterility of direct infusion systems using aseptic technique during clinical use. Multiple patient infusions from direct infusion systems, such as the portable PET patient infusion device and ${ }^{82} \mathrm{Rb}$ generators, are completed in ambient air environments with potential for contamination. Following specific device labeling for set-up, clinical use, storage, and disposal is a requirement. Guidance in USP $<825>$ includes instructions for setting up the infusion system; cleaning and disinfecting vials, eluent bag, and infusion device; as well as observing a BUD of $10 \mathrm{~h}$ for aseptic handling in an ambient air environment (4). Only 1 puncture of the sterile container is allowed. If there is a problem with the infusion device, the sterile container, along with its contents, must be discarded.

\section{USP $<\mathbf{8 2 5}>$ IMPLEMENTATION TIMELINE}

USP general chapter $<825>$ was published on June 1 , 2019 , with the goal of being implemented 6 mo later on December 1, 2019. After publication of the new standard, the USP received 2 appeals, which delayed its implementation. The first appeal questioned the standards requiring compounding using sterile substances, assignment of BUDs, and compounding of radiopharmaceuticals available in an FDA-approved format and was denied after consideration by the responsible USP expert committee (7). The second appeal, which requested deviations from the proposed framework and BUD provisions of USP $<825>$, was granted an official USP appeals hearing on January 21, 2020, and was denied. The USP will publish a new official approval date, followed by another 6-mo period for implementation (8). Until then, the nuclear medicine community continues to compound, prepare, and dispense sterile radiopharmaceuticals under the guidance of the 2008 revision of USP $<797>$ (9).

\section{CONCLUSION}

Since 2004, nuclear medicine technologists' activities associated with the sterile preparation and clinical use of radiopharmaceuticals have been subject to inspection by the Joint Commission, the Centers for Medicare and Medicaid Services, and other regulatory bodies under USP $<797>$ standards. USP $<825>$ was developed to provide clear and effective standards that meet patient and practitioner requirements specifically for compounded sterile and nonsterile radiopharmaceuticals. The primary objective for the development of USP $<825>$ is an improved understanding of the expectations related to sterile preparation of radioactive drugs. Regarding the traditional practice of compounding sterile radiopharmaceuticals using radionuclide generators, radiopharmaceutical manufacturer's kits, bulk chemicals, and radionuclide supplies, the requirements of USP $<825>$ are not different from those of the USP $<797>$ standards. There were only minor changes in USP $<825>$ to personnel qualifications, training, hand hygiene, garbing, facilities and engineering controls, cleaning and disinfecting, microbiologic air and surface monitoring, and records maintenance to accommodate the required 
observation of radiation safety practices. USP $<825>$ includes significant changes to accommodate the preparation and dispensing of sterile radiopharmaceuticals typically completed by nuclear medicine technologists to meet individual patient needs.

\section{DISCLOSURE}

No potential conflict of interest relevant to this article was reported.

\section{REFERENCES}

1. Chapter $<797>$ pharmaceutical compounding: sterile preparations. In: USP-NF. Rockville, MD: United States Pharmacopeial Convention; 2016.

2. Nuclear medicine technologist scope of practice and performance standards. J Nucl Med Technol. 2017;45:53-64.

3. USP public standards for compounded sterile radiopharmaceuticals. recommendations from SNMMI. SNMMI file on Amazon S3 website. http://snmmi.
files.cms-plus.com/SNMMI-USP-Recommendations-Final_2016.pdf. Accessed May $8,2020$.

4. Chapter $<825>$ radiopharmaceuticals: preparation, compounding, dispensing, and repackaging. In: USP-NF. Rockville, MD: United States Pharmacopeial Convention; 2019.

5. UltraTag ${ }^{\mathrm{TM}} \mathrm{RBC}$ [prescribing information]. Curium website. https://www.curiumpharma. com/wp-content/uploads/2018/05/Ultratag-RBC-Insert-122018.pdf. Published 2018. Accessed May 8, 2020.

6. The ASRT practice standards for medical imaging and radiation therapy. ASRT website. https://www.asit.org/main/standards-and-regulations/professional-practice/ practice-standards-online. Accessed May 8, 2020.

7. Decision on appeal to USP $<825>$. USP website. https://www.usp.org/sites/default/ files/usp/document/health-quality-safety/usp-decision-on-appeals-825-factsheet.pdf. Published September 13, 2019. Accessed May 8, 2020.

8. Role and applicability of USP general chapter $<825>$ related to radiopharmaceuticals. USP website. https://www.usp.org/sites/default/files/usp/document/ourwork/compounding/applicability-gc825-fs.pdf. Published March 12, 2020. Accessed May 8, 2020.

9. USP general chapters $<795>,<797>,<800>$, and $<825>$. USP-NF website. https://www.uspnf.com/notices/compounding-chapters-postponement?_ga=2.29474876. 2025631567.1575900869-4464392.1569260113. Published September 23, 2019. Accessed May 8, 2020. 